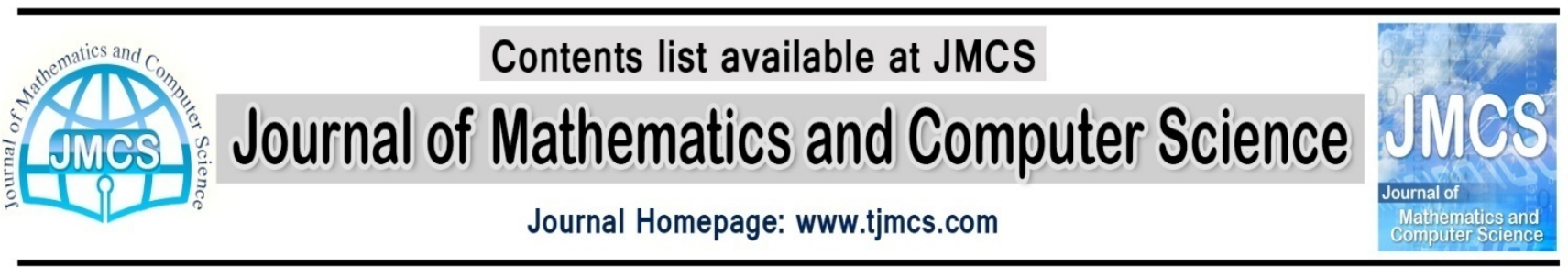

\title{
Another proof for the existence of dominated splitting for robustly ergodic diffeomorphisms
}

\author{
Alireza Zamani bahabadi \\ Department of Mathematics Ferdowsi University of Mashhad, Mashhad, Iran \\ zamany@um.ac.ir
}

Article history:

Received: December 2012

Accepted : January 2013

Available online: January 2013

\begin{abstract}
In this paper we show that any robustly ergodic system admits a dominated splitting without using pasting lemma for conservative diffeomorphisms.
\end{abstract}

Keywords: Ergodic, dominated splitting, conservative diffeomorphism.

\section{Introduction}

We shall address here the question of how the important concepts of robust ergodicity and dominated splitting are related. Ali Tahzibi in [4] studied the relation between robust transitivity and robust ergodicity for conservative diffeomorphism. This is well known that robustly transitive systems admit a dominated splitting.

Ali Tahzibi mentioned an interesting question for robustly ergodicdiffeomorphism as in the following:

1.1. Question Is it true that any $C^{1}$ robustly ergodic conservate diffeomorphism admits dominated splitting?

Using pasting lemma for conservative diffeomorphism, A.Arbieto and C.Matheus in [1] showed that robustly transitive conservative diffeomorphisms admit a non-trivial dominated splitting defined on the whole $M$. So robustly ergodic diffeomorphisms admit a dominated splitting. 
In this paper we give another proof of the existence of dominated splitting for robustly ergodic diffeomorphisms without using pasting lemma for conservative diffeomorphisms.

A $D f$-invariant splitting $E \oplus F$ of TM is called dominated splitting if the fibers of the bundles have constant dimension on whole manifold and there is $\lambda<1$ such that:

$$
\left.\mathrm{P} D f\right|_{E_{x}} \mathrm{P} . \mathrm{P} D f^{-1} \mid F(f(x)) \mathrm{P} \leq \lambda \forall x \in M \text {. }
$$

Let $\operatorname{Diff}_{m}^{1}(M)$ denote the set of diffeomorphisms which preserve the Lebesgue measure $m$ induced by the Riemannian metric. We endow this space with the $C^{1}$-topology.

Let $\operatorname{Diff}_{m}^{1+\alpha}(M)$ denote the subset of $\operatorname{Diff} f_{m}^{1}(M)$ for which the derivative is $\alpha$-Holder continuous and put $\operatorname{Diff}_{m}^{1+}(M)=\bigcup_{\alpha>0} \operatorname{Diff}_{m}^{1+\alpha}$.

\subsection{Theorem (Main theorem)}

Let $f \in \operatorname{Diff} f_{m}^{1}(M)$ be robustly ergodic. Then $f$ admits a dominated splitting.

For the prove of the above theorem we need some notions and lemmas.

For a periodic point $p$ of $f \in \operatorname{Diff}_{m}^{1}(M)$, we assume that the eigenvalues of $D f^{\pi(p)}$ are $\left\{\lambda_{1}, \lambda_{2}, \cdots, \lambda_{d}\right\}$ for witch

$$
\left|\lambda_{1}\right| \leq\left|\lambda_{2}\right| \leq \cdots \leq\left|\lambda_{d}\right|
$$

We say that $p$ is an almost source if $\left|\lambda_{1}\right|=1 ; p$ is an almost sink if $\left|\lambda_{d}\right|=1$.

\subsection{Lemma}

Let $f \in \operatorname{Diff}_{m}^{1}(M)$ be robustly ergodic. Then $f$ has neither almost sinks nor almost sources.

For the proof of the above lemma we need to conservatve version of Franks lemma [2].

\subsection{Proposition (Conservative version of Franks Lemma)}

Let $f$ be a diffeomorphism preserving a smooth measure $m, p$ be a periodic point. Assume that $B$ is a conservative $\varepsilon$-perturbation of $D f$ along the orbit of $p$. Then for every neighborhood $V$ of the orbit of $p$ there is a $C^{1}$-perturbation $h \in C^{1+\alpha}$ preserving $m$ and coinciding with $f$ on the orbit of $p$ and out of $V$, such that $D h$ is equal to $B$ on the orbit of $p$.

\section{Proof (lemma 1.3)}


Since $f$ is robustly ergodic, then there is a neighbourhood $U_{f}$ of $f$ in Diff $f_{m}^{1+}$ such that every $g \in U_{f}$ is ergodic. Assume $p$ is an almost sink for $f$. By conservative version of Franks lemma there is a $g \in U_{f} \cap \operatorname{Diff}_{m}^{1+\alpha}(M)$ such that $p$ is a sink for $g$. which is a contradiction because conservative systems have no sink. This completes the proof of lemma.

\subsection{Lemma}

For any $\varepsilon>0$ and for any neighbourhood $U$ of $f$ in Diff ${ }_{m}^{1}(M)$, there is a periodic point $p$ of $g \in U \cap \operatorname{Diff}_{m}^{1+}(M)$ such that $d_{H}\left(\operatorname{Orb}_{g}(p), M\right)<\varepsilon$.

For the proof of the above lemma we need the ergodic closing lemma as following;

\section{Theorem B(Ergodic closing lemma).}

Consider a diffeomorphism $f$ preserving a smooth volume $m$. Then there is an $f$-invariant set $\Sigma(f)$, such that:

(1) $\mu(\Sigma(f))=1$ for any invariant probability measure $\mu$.

(2) For every $x \in \Sigma(f)$ and $\varepsilon>0$ there is a $C^{1}$-perturbation $g \in C^{1+\alpha}$ preserving $m$ such that $x$ is a periodic point of $g$ and $d\left(f^{i}(x), g^{i}(x)\right)<\varepsilon$ for all $i \in\left[0, \pi_{g}(x)\right], \pi_{g}(x)$ is the period of $x$ with respect to $g$.

Any $x \in \Sigma(f)$ is called a well closable point.

\section{Proof. (lemma 1.5)}

Since $f$ is transitive, there is $x \in M$ such that $\omega(x)=M$. There is $N_{1} \in \mathrm{N}$ such that $d_{H}\left(\left\{x, f(x), \cdots, f^{N_{1}}(x)\right\}, \operatorname{Orb}_{f}(x)\right)<\frac{\varepsilon}{4}$, since $M$ is compact. Since $\omega(x)=M$, there is $N_{2}>N_{1}$ such that $d_{H}\left(\left\{x, \cdots, f^{n}(x)\right\}, M\right)<\frac{\varepsilon}{4}$ for any $n>N_{2}$. Choose $\delta>0$ such that if for $y \in M$, $d(x, y)<\delta$ then $d\left(f^{i}(x), f^{i}(y)\right)<\frac{\varepsilon}{4}$ for $i=0, \cdots, N_{2}+1$. By Ergodic closing Lemma, since $m\left(N_{\frac{\delta}{2}}(x)\right)>0$, then $\sum(f) \cap N_{\frac{\delta}{2}}(x) \neq \varnothing$. Let $p \in N_{\frac{\delta}{2}}(x)$ be a well closable point. For $\delta>0$ there is a $C^{1}$-perturbation $g \in \operatorname{Diff}_{m}^{1+\alpha}(M)$ such that $p$ is a periodic point of $g$ and $d\left(f^{i}(p), g^{i}(p)\right)<\frac{\delta}{2}$ for all $i \in\left[0, \pi_{g}(p)\right]$, where $\pi_{g}(p)>N_{2}$ is the period of $p$ with respect to $g$. Since $d(x, p)<\delta$ then, $d\left(f^{i}(x), f^{i}(p)\right)<\frac{\varepsilon}{4}$ for $i=0, \cdots, N_{2}+1$. So $d\left(f^{i}(x), g^{i}(p)\right)<\frac{\varepsilon}{2}$ for $i=0, \cdots, N_{2}+1$. By the above process we have $d_{H}\left(\operatorname{Orb}_{g}(p), M\right)<\varepsilon$. 
As a corollary,

\subsection{Corollary}

There are a sequence of diffeomorphisms $\left\{f_{n}\right\}$ in Diff ${ }_{m}^{1+}(M)$ and a sequence of point $\left\{p_{n}\right\}$ such that $p_{n}$ is a periodic point of $f_{n} \lim f_{n}=f$ and $\lim \operatorname{Orb}\left(p_{n}\right)=M$.

For the proof of the main theorem we also need the following Lemma in [2, Lemma 1.4].

\subsection{Lemma}

Give $c>0$ and $A \in(0,1)$. If there is a sequence of diffeomorphisms $\left\{f_{n}\right\}$ and a sequence of compact sets $\left\{\Lambda_{n}\right\}$ such that $\Lambda_{n}$ is a compact invariant set of $f_{n}$ and $\Lambda_{n}$ admits a $(c, A)$-dominated splitting of index $i$ with respect to $f_{n}$, then if $\Lambda=\lim _{n \rightarrow \infty} \Lambda_{n}$ exists, then $\Lambda$ admits a $(c, \lambda)$-dominated splitting of index $i$ with respect to $f$.

\section{Proof. (main theorem1.2)}

Let $\left\{p_{n}\right\}$ be in the above corollary.

Let $\Sigma=\coprod_{n \in \mathbb{N}}\left\{p_{n}, f\left(p_{n}\right), \cdots, f^{\pi\left(p_{n}\right)-1}\left(p_{n}\right)\right\}$. One can define a natural d-dimensional vector bundle $E$ on $\Sigma$ as following:

for any $x \in \Sigma$, the fiber on $x$ is $T_{x} M$. For any $i \in\left[0, \pi\left(p_{n}\right)-1 \cap N\right]$, we define $h\left(f_{n}^{i}\left(p_{n}\right)\right)=f_{n}^{i+1}\left(p_{n}\right)$ and $\left.A\right|_{E\left(f_{n}^{i}\left(p_{n}\right)\right)}=D f_{n}\left(f_{n}^{i}\left(p_{n}\right)\right)$. Thus $\mathrm{A}=(\Sigma, h, E, A)$ is a bounded large periodic systems as in [3]. Then by [3,Theorem 2.2] either there is an infinite subset $\Sigma^{\prime} \subset \Sigma$ which is invariant by $h$ such that the periodic linear cocycle $A^{\prime}=\left(\Sigma^{\prime}, h,\left.E\right|_{\Sigma^{\prime}}, A\right)$ admits a dominated splitting or there is a perturbation $\mathrm{B}$ of $A$ and an in finite invariant subset $\Sigma^{\prime}$ of $\Sigma$ such that for any $x \in \Sigma$, all eigenvalues of $B\left(h^{\pi(x)-1}(x)\right) \circ B\left(h^{\pi(x)-2}(x)\right) \circ \cdots \circ B(x)$ are real, with same modulus.

By Remark 7.2 in [2] we can consider the perturbation $A^{\prime}$ such that $\operatorname{det} A^{\prime}(x)=1$ for every $x \in \Sigma^{\prime}$. Then by proposition 1.4 we can translate the above statement for $\operatorname{Diff}_{m}^{1}(M)$.

either there are constant $c>0$ and $A \in(0,1)$ such that there is a $c, \lambda)$-dominated splitting on the orbit $\left\{p_{n}\right\}$; or there is a sequence of diffeomorphism $\left\{g_{n}\right\}$ in $\operatorname{Diff}_{m}^{1+}(M)$ such that $\lim _{n \rightarrow \infty} g_{n}=f$ and $\operatorname{Orb}_{f_{n}}\left(p_{n}\right)$ is also a periodic orbit of $g_{n}$ and all eigenvalues of $D g_{n}^{\pi\left(p_{n}\right)}\left(p_{n}\right)$ are all real, with same modulus. 
So by an perturbation there is an almost sink or an almost source for a $g \in \operatorname{Diff}_{m}^{1+}(M)$ near $f$ which contradicts to the fact that $f$ is robustly ergodic. Thus the second case of the above statement is false for $f$.

Now by corollary1.6 and letting $\Lambda_{n}=M$ in the Lemma1.7, the proof of the main theorem is complete.

Pengfel Zhang in [5] showed that if $f$ has a dominated splitting $T M=E \oplus F$, then $f$ can not be minimal. Recall that the map $f$ is said to be minimal if for each $x \in M$, the orbit $\mathrm{O}(x)=\left\{f^{n}(x): n \in Z\right\}$ is a dense subset in $M$. So we have the following corollary.

\subsection{Corollary}

If $f$ is robustly ergodic, then $f$ is not minimal.

\section{References}

[1] A. Arbieto, C. Matheus, A pating lemma and some applications for conservative systems,Ergodic Theory and Dynamical Systems. 27, 1399-1417 (2007).

[2] C. Bonatti, L. Diaz, E. Pujals, A $C^{1}$-generic dichotomy for diffeomorphisms: Weak forms of hyperbolicity or infinitely many sinks or sources, Ann. Math. 158, 355-418 (2003).

[3] C. Bonatti, N. Gourmelon and T. Vivier, Perturbations of the derivatives along periodic orbit, Ergodic Theory Dynam, systems, 26, 1307-1377 (2006).

[4] A Tahzibi, Robust transitivity almost robust ergodicity,Ergodic Theory and Dynamical Systems, 24:4:1261-1269 (2004).

[5] P. Zhang, A diffeomorphism with global dominated splitting can not be minimal. Proc. Am. Math. Soc., , 140: 589 - 593 (2012). 